\title{
Decoherence and Recoherence of Beam in Phase Space
}

\author{
Jicong Shi and Sho Ohnuma \\ Department of Physics, University of Houston, Houston, TX 77204-5506, USA
}

\section{Abstract}

Using the particle distribution in phase space, we introduce a new approach to study the decoherence and recoherence of a kicked beam when betatron tunes are far from any resonance. With this method, the decoherence and the recoherence of a beam can be analyzed easily in both 2-dimensional and 4-dimensional cases with any form of the tune spread. We have also studicd the decoherence of a beam initially trapped in a resonance island. Due to the tune modulation, the separatrix of island develops a chaotic layer and particles within the island can drift out of the island along the layer. Consequently, the beam decoheres gradually. The rate of decoherence is shown to be proportional to the modulation amplitude. As a function of the modulation frequency, it reaches a peak near the tune of trapped particles before decreasing to zero.

\section{DECOHERENCE IN THE NEAR-LINEAR REGIME}

In the presence of amplitude dependence of betatron tunes (tune spread), an off-centered beam will gradually dilute from a localized bunch to an annulus in transverse phase-space which results in a disappearance of coherent signal. Due to a finite energy spread, the beam may also oscillate between the localized bunch and the annulus which results in a periodic oscillation of the coherent signal. These decoherence and recoherence of a kicked beam have been studied experimentally as well as analytically $[1,2]$. Previous studies based on a single-particle picture were however limited to quadratic amplitude-dependence of the tunes. Since the decoherence and recoherence are basically multiparticle phenomena, a more suitable as well as easier description should be based on a study of the particle distribution in phase space. In this note, we shall introduce this approach to study the decoherence and recoherence. With our method, these phenomena can be analyzed in both 2 and 4-dimensional cases with any form of amplitude dependence of the tunes.

In general, the Hamiltonian of 4-dimensional betatron oscillations can be written as

$$
H=\vec{\nu} \cdot \vec{J}+U_{0}(\vec{J})+U(\vec{J}, \vec{\phi}, \theta),
$$

where $\vec{\nu}=\left(\nu_{1}, \nu_{2}\right)$ are betatron tunes, $\theta$ the independent variable which is the path length of the central orbit divided by the average machine radius. $(\vec{J}, \vec{\phi})=$

\footnotetext{
${ }^{1}$ Supported by TNRLC under award FCFY9221 and the U.S. Department of Energy under grant DE-FG05-87ER40374.
}

$\left(J_{1}, \phi_{1}, J_{2}, \phi_{2}\right)$ are action-angle variables:

$$
\begin{aligned}
& \sqrt{2 J_{k}} \cos \phi_{k}=z_{k} / \sqrt{\beta_{k}} \\
& \sqrt{2 J_{k}} \sin \phi_{k}=-\sqrt{\beta_{k}} z_{k}^{\prime}+\beta_{k}^{\prime} z_{k} /\left(2 \sqrt{\beta_{k}}\right),
\end{aligned}
$$

where $k=1,2$ and $\left(z_{1}, z_{2}\right)$ denote $(x, y) . U_{0}(\vec{J})+U(\vec{J}, \vec{\phi}, \theta)$ represents the nonlinear perturbation of which $U_{0}(\vec{J})$ depends on $\vec{J}$ only and $\langle U\rangle_{\vec{\phi}, \theta}=0$. The transverse distribution of the beam particle $f_{T}(\vec{J}, \vec{\phi}, \theta)$ satisfies the equation

$$
\frac{\partial f_{T}}{\partial \theta}=\left[H, f_{T}\right],
$$

where [] is the Poisson bracket. Since the time scale we are interested in is much shorter than the diffusion time scale, as betatron tunes are far from any major resonance, we consider $U_{0}$ only and $\mathrm{Eq}$. (4) is reduced to

$$
\frac{\partial f_{T}}{\partial \theta}+\left[\vec{\nu}+\frac{\partial U_{0}}{\partial \vec{J}}\right] \cdot \frac{\partial f_{T}}{\partial \vec{\phi}}=0
$$

The solution of this equation is easily found to be

$$
\begin{aligned}
f_{T}(\vec{J}, \vec{\phi}, \theta) & =f_{T}\left(\vec{J}, \vec{\phi}-\int_{0}^{\theta} \vec{\nu}(\tau) d \tau-\frac{\partial U_{0}}{\partial \vec{J}} \theta, 0\right) \\
& =f_{T}(\vec{J}, \vec{\psi}, 0)
\end{aligned}
$$

where $f_{T}(\vec{J}, \vec{\phi}, 0)$ is the initial distribution and

$$
\vec{\psi}=\vec{\phi}-\int_{0}^{\theta} \vec{\nu}(\tau) d \tau-\theta \frac{\partial U_{0}}{\partial \vec{J}}=\vec{\phi}-\vec{\chi} .
$$

If the synchrotron motion is assumed to be linear,

$$
\vec{\nu}=\vec{\nu}_{0}+\vec{\xi} \sqrt{J_{L} / \beta_{L}} \sin \left(\nu_{s} \theta+\phi_{L}\right),
$$

where $\vec{\xi}$ is chromaticity, $\left(J_{L}, \phi_{L}\right)$ the action-angle variable for synchrotron motion, and $\nu_{s}$ the synchrotron tune, and

$$
\vec{\chi}=\vec{\nu}_{0} \theta+\frac{2 \vec{\xi}}{\nu_{s}}\left(\frac{J_{L}}{\beta_{L}}\right)^{\frac{1}{2}} \sin \left(\frac{\nu_{s} \theta}{2}\right) \sin \left(\nu_{s} \theta+\phi_{L}\right)+\theta \frac{\partial U_{0}}{\partial \vec{J}} .
$$

Let $f_{L}\left(J_{L}, \phi_{L}\right)$ denote the beam particle distribution in the longitudinal phase-space. The beam centroid can be calculated from

$$
\begin{aligned}
& \left\langle\sqrt{2 J_{k}} e^{-i \phi_{k}}\right\rangle_{\vec{J}, \vec{\phi}} \\
& =\int \sqrt{2 J_{k}} e^{-i \phi_{k}} f_{T}(\vec{J}, \vec{\phi}, \theta) f_{L}\left(J_{L}, \phi_{L}\right) d \vec{J} d \vec{\phi} d J_{L} d \phi_{L}
\end{aligned}
$$




$$
\begin{aligned}
& =\int \sqrt{2 J_{k}} e^{-i \phi_{k}} f_{T}(\vec{J}, \vec{\phi}-\vec{\chi}, 0) f_{L}\left(J_{L}, \phi_{L}\right) d \vec{J} d \vec{\phi} d J_{L} d \phi_{L} \\
& =\int \sqrt{2 J_{k}} e^{-i\left(\phi_{k}+\chi_{k}\right)} f_{T}(\vec{J}, \vec{\phi}, 0) f_{L}\left(J_{L}, \phi_{L}\right) d \vec{J} d \vec{\phi} d J_{L} d \phi_{L} \\
& =e^{-i \nu_{0 k} \theta} \int \exp \left(-i a_{k} \sqrt{J_{L}} \sin \phi_{L}\right) f_{L}\left(J_{L}, \phi_{L}\right) d I_{L} d \phi_{L} \\
& \times \int \sqrt{2 J_{k}} \exp \left[-i\left(\phi_{k}+\theta \frac{\partial U_{0}}{\partial J_{k}}\right)\right] f_{T}(\vec{J}, \vec{\phi}, 0) d \vec{J} d \vec{\phi},(10)
\end{aligned}
$$

where $k=1,2$.

Assume that initially the beam has a Gaussian distribution with its centroid located at $\left(x, x^{\prime}, y, y^{\prime}\right)=$ $\left(z_{01}, z_{01}^{\prime}, z_{02}, z_{02}^{\prime}\right)$ in the transverse phase-space,

$$
f_{T}(\vec{J}, \vec{\phi}, 0)=f_{1}\left(J_{1}, \phi_{1}\right) f_{2}\left(J_{2}, \phi_{2}\right),
$$

where

$$
\begin{aligned}
& f_{k}\left(J_{k}, \phi_{k}\right)=\frac{1}{2 \pi \sigma_{k}^{2}} \\
& \times \exp \left\{-\frac{1}{\sigma_{k}^{2}}\left[J_{k}+J_{0 k}-2 \sqrt{J_{0 k} J_{k}} \cos \left(\phi_{k}-\phi_{0 k}\right)\right]\right\},
\end{aligned}
$$

and

$$
\begin{aligned}
& \sqrt{2 J_{0 k}} \cos \phi_{0 k}=z_{0 k} / \sqrt{\beta_{k}}, \\
& \sqrt{2 J_{0 k}} \sin \phi_{0 k}=-\sqrt{\beta_{k}} z_{0 k}^{\prime}+\beta_{k}^{\prime} z_{0 k} /\left(2 \sqrt{\beta_{k}}\right)
\end{aligned}
$$

with $k=1,2$. We also assume that the beam has a Gaussian distribution in the longitudinal phase-space,

$$
f_{L}\left(J_{L}, \phi_{L}\right)=\frac{1}{2 \pi \sigma_{L}^{2}} \exp \left(-\frac{J_{L}}{\sigma_{L}^{2}}\right) .
$$

Substitution of Eqs. (11)-(15) into Eq. (10) yields

$$
\begin{gathered}
\left\langle\sqrt{2 J_{k}} e^{-i \phi_{k}}\right\rangle_{\vec{J}, \vec{\phi}}=\exp \left[-i\left(\phi_{0 k}+\nu_{0 k} \theta\right)-\frac{J_{0 k}}{\sigma_{k}^{2}}-\frac{J_{0 j}}{\sigma_{j}^{2}}\right] \\
\times \exp \left[-\frac{\xi_{k}^{2} \sigma_{L}^{2}}{\nu_{s}^{2} \beta_{L}} \sin ^{2}\left(\frac{1}{2} \nu_{s} \theta\right)\right] \\
\times \frac{1}{\sigma_{k}^{2} \sigma_{j}^{2}} \int d J_{j} I_{0}\left(\frac{2 \sqrt{J_{0 j} J_{j}}}{\sigma_{j}^{2}}\right) \exp \left(-\frac{J_{j}}{\sigma_{j}^{2}}\right) \\
\times \int d J_{k} \sqrt{2 J_{k}} I_{1}\left(\frac{2 \sqrt{J_{0 k} J_{k}}}{\sigma_{k}^{2}}\right) \exp \left(-\frac{J_{k}}{\sigma_{k}^{2}}+i \theta \frac{\partial U_{0}}{\partial J_{k}}\right),
\end{gathered}
$$

where $I_{0}$ and $I_{1}$ are modified Bessel functions of order 0 and 1 respectively. $(k, j)$ is any permutation of 1 and 2 . After knowing the amplitude dependence of the betatron tunes, the time evolution of beam centroid can be obtained by integrating $J_{k}$ and $J_{j}$ in Eq. (16) analytically or numerically.

If we consider only the lowest-order amplitude dependence of the tunes:

$$
U_{0}=\frac{1}{2} b_{1} J_{1}^{2}+b_{3} J_{1} J_{2}+\frac{1}{2} b_{2} J_{2}^{2}
$$

the beam centroid is found from Eq. (16) as

$$
\begin{gathered}
\left\langle\sqrt{2 J_{k}} e^{-i \phi_{k}}\right\rangle_{\vec{J}, \vec{\phi}}=\frac{\sqrt{2 J_{0 k}}}{\left[1+\left(\sigma_{k}^{2} b_{k} \theta\right)^{2}\right]\left[1+\left(\sigma_{j}^{2} b_{3} \theta\right)^{2}\right]^{1 / 2}} \\
\times \exp \left\{-\frac{J_{0 k}\left(\sigma_{k} b_{k} \theta\right)^{2}}{1+\left(\sigma_{k}^{2} b_{k} \theta\right)^{2}}-\frac{J_{0 j}\left(\sigma_{j} b_{3} \theta\right)^{2}}{1+\left(\sigma_{j}^{2} b_{3} \theta\right)^{2}}\right\} \\
\times \exp \left\{-\frac{\xi_{k}^{2} \sigma_{L}^{2}}{\nu_{s}^{2} \beta_{L}} \sin ^{2}\left(\frac{1}{2} \nu_{s} \theta\right)\right\} \exp \left(-i \phi_{c k}\right),
\end{gathered}
$$

where

$$
\begin{aligned}
\phi_{c k}(\theta)= & \phi_{0 k}+\nu_{0 k} \theta+2 \tan ^{-1}\left(b_{k} \sigma_{k}^{2} \theta\right)+\tan ^{-1}\left(b_{3} \sigma_{j}^{2} \theta\right) \\
& +\frac{J_{0 k} b_{k} \theta}{1+\left(\sigma_{k}^{2} b_{k} \theta\right)^{2}}+\frac{J_{0 j} b_{3} \theta}{1+\left(\sigma_{j}^{2} b_{3} \theta\right)^{2}}
\end{aligned}
$$

and $(k, j)$ is any permutation of 1 and 2 . The amplitudes of the beam centroid are thus

$$
\begin{aligned}
A_{k}(\theta)= & \left|\left\langle\sqrt{2 J_{k}} e^{-i \phi_{k}}\right\rangle_{\vec{J}, \vec{\phi}}\right| \\
= & \frac{\sqrt{2 J_{0 k}}}{\left[1+\left(\sigma_{k}^{2} b_{k} \theta\right)^{2}\right]\left[1+\left(\sigma_{j}^{2} b_{3} \theta\right)^{2}\right]^{1 / 2}} \\
& \times \exp \left\{-\frac{J_{0 k}\left(\sigma_{k} b_{k} \theta\right)^{2}}{1+\left(\sigma_{k}^{2} b_{k} \theta\right)^{2}}-\frac{J_{0 j}\left(\sigma_{j} b_{3} \theta\right)^{2}}{1+\left(\sigma_{j}^{2} b_{3} \theta\right)^{2}}\right\} \\
& \times \exp \left\{-\frac{\xi_{k}^{2} \sigma_{L}^{2}}{\nu_{s}^{2} \beta_{L}} \sin ^{2}\left(\frac{1}{2} \nu_{s} \theta\right)\right\} .
\end{aligned}
$$

If $\partial^{2} U_{0} / \partial J_{j} \partial J_{k}=0$, i.e. the betatron tunes are independent of $\vec{J}$, we have

$$
A_{k}(\theta) / A_{k}(0)=\exp \left\{-\frac{\xi_{k}^{2} \sigma_{L}^{2}}{\nu_{s}^{2} \beta_{L}} \sin ^{2}\left(\frac{1}{2} \nu_{s} \theta\right)\right\} .
$$

Eq. (21) shows that the coherent signal slowly oscillates with the frequence of the tune modulation $\nu_{s}$. The smallest coherent signal occurs at $1 /\left(2 \nu_{s}\right)$ turns with

$$
A_{k}\left(\frac{1}{2 \nu_{s}}\right) / A_{k}(0)=\exp \left\{-\frac{\xi_{k}^{2} \sigma_{L}^{2}}{\nu_{s}^{2} \beta_{L}}\right\} \text {. }
$$

After each $1 / \nu_{s}$ turns a coherent signal with original magnitude reappears.

For $\partial^{2} U_{0} / \partial J_{j} \partial J_{k} \neq 0$, Eq. (20) describes the decrease of magnitude of the coherent signal due to a finite tune spread. It shows that:

1. The rate of this decrease is independence of $\nu_{s}$.

2. The larger the amplitude dependence of the tunes and the transverse beam size, the larger the rate.

3. Since the oscillation part of $A_{k}$ has a period of $1 / \nu_{s}$ turns, for

$$
\frac{\sigma_{k}^{2}}{\nu_{s}}\left|\frac{\partial^{2} U_{0}}{\partial J_{k}^{2}}\right| \ll<1, \text { and } \frac{\sigma_{j}^{2}}{\nu_{s}}\left|\frac{\partial^{2} U_{0}}{\partial J_{j} \partial J_{k}}\right| \ll<1,
$$


the first minimum of $A_{k}$ is around $\theta=\pi / \nu_{s}$ and the next maximum is around $\theta=2 \pi / \nu_{s}$. The ratio of the next maximum and first minimum is

$$
\begin{gathered}
\frac{A_{k, \max }}{A_{k, \min }}=\frac{\left[\nu_{s}^{2}+\left(\pi \sigma_{k}^{2} b_{k}\right)^{2}\right]\left[\nu_{s}^{2}+\left(\pi \sigma_{j}^{2} b_{3}\right)^{2}\right]^{1 / 2}}{\left[\nu_{s}^{2}+\left(2 \pi \sigma_{k}^{2} b_{k}\right)^{2}\right]\left[\nu_{s}^{2}+\left(2 \pi \sigma_{j}^{2} b_{3}\right)^{2}\right]^{1 / 2}} \\
\quad \times \exp \left\{-\frac{3 J_{0 k}\left(\pi \nu_{s} \sigma_{k} b_{k}\right)^{2}}{\left[\nu_{s}^{2}+\left(2 \pi \sigma_{k}^{2} b_{k}\right)^{2}\right]\left[\nu_{s}^{2}+\left(\pi \sigma_{k}^{2} b_{k}\right)^{2}\right]}\right. \\
\left.-\frac{3 J_{0 j}\left(\pi \nu_{s} \sigma_{j} b_{3}\right)^{2}}{\left[\nu_{s}^{2}+\left(2 \pi \sigma_{j}^{2} b_{3}\right)^{2}\right]\left[\nu_{s}^{2}+\left(\pi \sigma_{j}^{2} b_{3}\right)^{2}\right]}+\frac{\xi_{k}^{2} \sigma_{L}^{2}}{\nu_{s}^{2} \beta_{L}}\right\} .
\end{gathered}
$$

If this ratio is larger than 1 , a weaker coherent signal will reappear after $1 / \nu_{s}$ turns.

\section{DECOHERENCE OF BEAM IN A RESONANCE ISLAND}

Consider a beam kicked into a resonance island. If this resonance is isolated, and there is no tune modulation, the beam will be trapped inside the island "forever". With a tune modulation, the separatrix of island develops a chaotic layer. Particles within this chaotic layer will eventually drift out of the island along the layer. Consequently, the beam decoheres gradually. For a beam kicked into the neighborhood of the chaotic layer (part of beam overlap with the layer), more particles will drift out of the island once the layer becomes wider. If the drifting speed of particles is assumed to be uniform within the chaotic layer, the rate of decoherence of the beam is proportional to the width of the chaotic layer. Therefore, by estimating this width, the decoherence rate of a beam in the island can be understood at least semi-quantitatively. Here we only consider the motion in $x-x^{\prime}$ plane.

As we consider a $n$ th-order resonance, $n \nu_{0}=k$, the Hamiltonian can be written as

$$
H_{0}=\delta \nu J+U_{0}(J)+U_{1}(J) \cos \left(n \phi+\phi_{0}\right),
$$

where $\delta \nu=\nu-\nu_{0}, \phi_{0}$ is a phase constant, and $(J, \phi)$ is the action-angle variable of $\left(x, x^{\prime}\right)$. The tune modulation $\delta \nu=\delta \nu_{0}+D \cos \left(\nu_{s} \theta\right)$ is treated as a periodic perturbation,

$$
\begin{gathered}
H=\left[\delta \nu_{0}+D \cos \left(\nu_{s} \theta\right)\right] J+U_{0}(J)+U_{1}(J) \cos \left(n \phi+\phi_{0}\right) \\
=H_{0}+J D \cos \left(\nu_{s} \theta\right)
\end{gathered}
$$

Near the resonance, the Hamiltonian can be expanded with $\left(J-J_{r}\right)$ where $J_{r}$ is the value of $J$ for the resonance. Let $t=n \theta, r=U_{0}^{\prime \prime}\left(J_{r}\right)\left(J-J_{r}\right), \omega_{0}^{2}=-U_{0}^{\prime \prime}\left(J_{r}\right) U_{1}\left(J_{r}\right), \psi=$ $n \phi+\phi_{0}$, and $r_{r}=J_{r} U_{0}^{\prime \prime}\left(J_{r}\right)$, the new Hamiltonian is then

$$
\begin{aligned}
\tilde{H}(r, \psi, t) & =\frac{1}{2} r^{2}-\omega_{0}^{2} \cos \psi+D\left(r_{r}+r\right) \cos \left(\frac{\nu_{s}}{n} t\right) \\
& =\tilde{H}_{0}+D\left(r_{r}+r\right) \cos \left(\frac{\nu_{s}}{n} t\right) .
\end{aligned}
$$

This is the Hamiltonian for the pendulum with a periodic driving force. The motion in a vicinity of the separatrix can be described by the whisker map [3],

$$
\left\{\begin{array}{l}
w_{n+1}=w_{n}+W \sin \psi_{n} \\
\psi_{n+1}=\psi_{n}+\lambda \ln \left(32 /\left|w_{n+1}\right|\right)
\end{array}\right.
$$

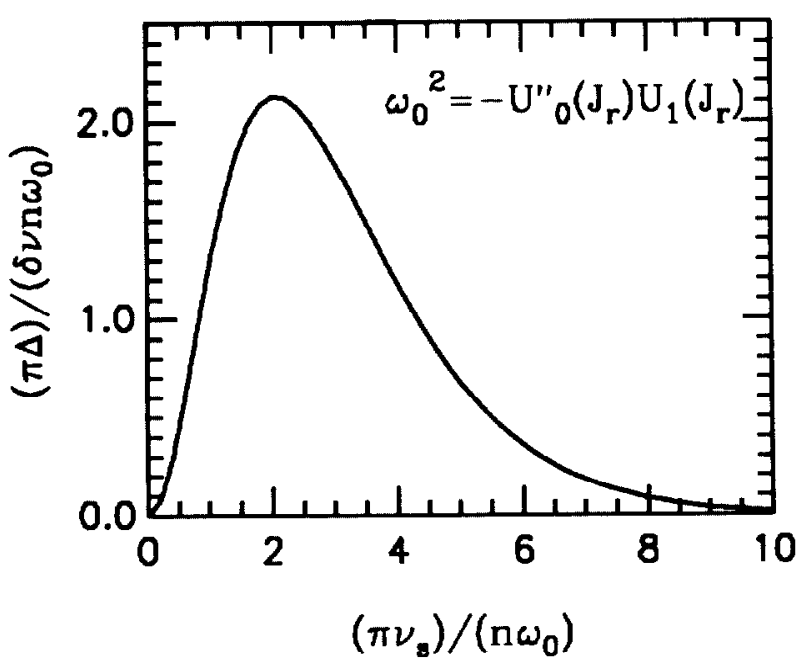

Figure 1: $\Delta$ as a function of $\nu_{s}$

where

$$
\begin{gathered}
w_{n}=\frac{\tilde{H}_{0}-\omega_{0}^{2}}{\omega_{0}^{2}}, \quad \lambda=\frac{\nu_{s}}{\omega_{0}}, \\
W=\frac{2 \pi \nu_{s} D}{n \omega_{0}^{2} \cosh \left[\pi \nu_{s} /\left(n \omega_{0}\right)\right]} .
\end{gathered}
$$

The width of the chaotic layer is [3]

$$
\Delta=\omega_{0}^{2} \lambda W=\frac{2 \pi \nu_{s}^{2} D}{n \omega_{0} \cosh \left[\pi \nu_{s} /\left(n \omega_{0}\right)\right]}
$$

Eq. (29) shows that :

1. The width of the chaotic layer, that is, the rate of decoherence is proportional to the modulation depth $D$.

2. $\Delta$ takes the maximum value at the modulation tune $\nu_{s}^{m}$ which is the solution of the equation,

$$
\begin{gathered}
\frac{\pi \nu_{s}^{m}}{n \omega_{0}}=2 \operatorname{coth}\left(\frac{\pi \nu_{s}^{m}}{n \omega_{0}}\right) . \\
\nu_{s}^{m} \simeq \frac{2 n}{\pi} \omega_{0}=\frac{2 n}{\pi}\left|U_{0}^{\prime \prime}\left(J_{r}\right) U_{1}\left(J_{r}\right)\right|^{\frac{1}{2}} .
\end{gathered}
$$

3. For $\nu_{s}<<1, \Delta \propto \nu_{s}^{2}$. For $\nu_{s} \rightarrow \infty, \Delta \longrightarrow 0$ (see Fig. $1)$.

\section{REFERENCES}

[1 ] F. Willeke, Fermilab Report TM-1309, (1985).

[2] R. E. Meller et al, SSCL Report SSC-N-360, (1987).

[3 ] Chirikov, B. V., Phys. Rep. 52, 265(1979). 